\title{
Social inequalities in wheezing in children: findings from the UK Millennium Cohort Study
}

\author{
David C. Taylor-Robinson ${ }^{1,2}$, Anna Pearce ${ }^{2}$, Margaret Whitehead ${ }^{1}$, \\ Rosalind Smyth ${ }^{2}$ and Catherine Law ${ }^{2}$
}

Affiliations:

${ }^{1}$ Dept of Public Health and Policy, University of Liverpool, Liverpool, UK.

${ }^{2}$ UCL Institute of Child Health, London, UK.

\section{Correspondence:}

David C. Taylor-Robinson, Dept of Public Health and Policy, Whelan Building, University of Liverpool, Liverpool, L69 3GB, UK.

E-mail: dctraliv.ac.uk

ABSTRACT Wheezing in childhood is socially patterned, but it is unclear what factors explain the social differences.

Regression analysis of the UK Millennium Cohort Study, based on 11141 singleton children who participated at ages 9 months and 3,5 and 7 years. Relative risk ratios (RRR) for early and persistent/ relapsing wheeze were estimated using multinomial regression, according to measures of socioeconomic circumstances. Maternal, antenatal and early-life characteristics were assessed as potential mediators.

Children of mothers with no educational qualifications were more likely to have both wheeze types, compared to children of mothers with degree-level qualifications (RRR 1.53, 95\% CI 1.26-1.86 for early wheeze; 1.32 95\% CI 1.04-1.67 for persistent/relapsing wheeze). Controlling for maternal age, smoking during pregnancy and breastfeeding removed the elevated risk of wheezing. Male sex, maternal age, body mass index, atopy, smoking during pregnancy, preterm birth, breastfeeding, exposure to other children and furry pets were independently associated with wheezing, but the pattern of association varied between wheezing types.

In this representative UK cohort, adjustment for maternal smoking during pregnancy and breastfeeding removed the socioeconomic inequalities in common wheezing phenotypes. Policies to reduce the social gradient in these risk factors may reduce inequalities in wheezing and asthma.

@ERSpublications

Increased wheeze in disadvantaged children removed by adjusting for smoking in pregnancy and lower breastfeeding rate http://ow.ly/TLLD8

This article has supplementary material available from erj.ersjournals.com

Received: March 242015 | Accepted after revision: Oct 13 2015 | First published online: Dec 172015

Support statement: D.C. Taylor-Robinson (G0802448) and A. Pearce are funded by Medical Research Council Population Health Scientist fellowships (MR/J012351/1). All researchers were independent of the funders and the funders played no part in the study design, analysis or interpretation of the data, writing of the report or the decision to submit for publication. Funding information for this articlehas been deposited with FundRef.

Conflict of interest: Disclosures can be found alongside the online version of this article at erj.ersjournals.com

Copyright OERS 2016. ERJ Open articles are open access and distributed under the terms of the Creative Commons Attribution Non-Commercial Licence 4.0. 


\section{Introduction}

Asthma is one of the most common chronic diseases of childhood, affecting about one in five children in the UK $[1,2]$. It is an important cause of avoidable hospital admissions in children, can limit a child's daily life, social activities and school performance, and can impact on parental employment [3]. Estimates suggest that the National Health Service spends approximately one billion GBP a year treating and caring for people with asthma, with the estimated annual cost of treating a child with asthma being higher than the cost per adult with asthma [4]. It is therefore important to identify modifiable risk factors for asthma that are amenable to public health intervention.

Lower socioeconomic position is associated with higher asthma and wheezing prevalence [5], but it remains unclear what factors explain the social patterning of childhood asthma. A better understanding of the role of early-life exposures is imperative to developing interventions to address the disproportionate burden of disease suffered by more disadvantaged populations. Furthermore, few studies have studied inequalities in different longitudinal wheezing patterns in children [5]. Asthma comprises a range of heterogeneous phenotypes that differ in presentation, aetiology and pathophysiology. Distinct patterns can be identified in childhood wheezing illness, and accurate classification of childhood wheeze phenotypes and an understanding of their natural history are important to design interventions and improve outcomes [6]. Commonly described phenotypes in early infancy and childhood are early transient wheezing, intermediate-onset wheezing and persistent wheezing $[7,8]$. Transient wheezing is well characterised, with onset of wheezing within the first year of life, resolution of symptoms by mid-childhood and no lasting subsequent effects on pulmonary function. By contrast, all the other phenotypes can lead to persistent symptoms in adulthood [9]. Identifying risk factors for different patterns of wheezing should aid understanding of how inequalities in wheezing arise and persist and how they might be tackled to improve health in childhood and prevent wheezing in adulthood.

Using contemporary and nationally representative data from the UK, the aim of this study was to explore how socioeconomic circumstances (SEC) influence longitudinal wheezing patterns. Our objectives were to identify early-life risk factors for longitudinal wheezing patterns and to explore how early-life risk factors explain any differences in wheezing by SECs.

\section{Methods}

Design, setting and data source

We analysed data from the Millennium Cohort Study (MCS), a nationally representative sample of children born in the UK between September 2000 and January 2002. Data were downloaded from the UK Data Archive in 2014. The study over-samples children living in disadvantaged areas and those with high proportions of ethnic minority groups by means of a stratified clustered sampling design [10]. Trained interviewers carried out home-based survey interviews with the main respondents (usually the mother) and their partners. Further information on the cohort and sampling design can be found online (www.cls.ioe.ac.uk/mcs). 18296 children were recruited at the age of 9 months. This study uses data collected on 11418 children (62\% of the total cohort) when they were aged 9 months and 3, 5 and 7 years old. The analysis did not require additional ethical approval.

\section{Outcome measures}

At 3, 5 and 7 years mothers were asked whether their child had any "wheezing in the last 12 months". This question was taken from the International Study of Asthma and Allergies in Childhood (ISAAC) core questionnaire for asthma [11]. This validated instrument has been used widely to measure childhood asthma and wheezing illnesses. Mothers were also asked at 9 months if their child had been "taken to a health professional for wheeze".

We included all children with responses to these questions, and categorised them according to whether they had wheezing symptoms or not at each time point. We then categorised these longitudinal binary trajectories, aiming to separate early wheezing from patterns of wheezing that persist, informed by the classification used by MARTINEZ and colleagues [12-14], where children with early transient wheezing were symptom-free at the age of 6 years, and two more recent large epidemiological studies $[15,16]$. In the latter studies the early wheezing phenotypes had remitted by the age of 7 years. We categorised children as having "early remitting" wheeze (wheezing at any point up to 5 years of age, but not at age 7 years); "persistent/relapsing" wheeze (patterns with wheezing present at the age of 7 years to capture persistent and late-onset wheezing); and "transient/intermittent" wheeze (not fitting either pattern). The main analyses focus on two phenotypes: early remitting wheeze, the most common phenotype identified in other studies, and persistent/relapsing wheeze, with never wheezing as the reference group (online supplementary material). 


\section{Early-life risk factors}

The primary exposure was highest maternal qualification achieved (National Vocational Qualifications or equivalent groups), used as a fixed measure of SECs at birth. Other factors potentially associated with asthma risk included the following [1, 8, 17-19]. 1) Demographic characteristics: sex of child and ethnicity; 2) maternal characteristics fixed at birth: age at birth of child, maternal history of atopy (none, asthma or eczema, asthma and eczema) and maternal prepregnancy body mass index (BMI) (underweight, normal, overweight, obese or morbidly obese); 3) perinatal factors and exposures during pregnancy: maternal smoking (never, smoked before pregnancy, smoked before and during pregnancy) to capture timing, amount smoked during pregnancy (never, $1-9$ cigarettes day $^{-1}, 10-19$ cigarettes $\cdot$ day $^{-1}$ and $\geqslant 20$ cigarettes $^{-d_{a y}{ }^{-1}}$ ) to assess doseresponse effects, Caesarean section (yes or no), preterm birth $<37$ weeks gestation (yes or no) and low birthweight $<2.5 \mathrm{~kg}$ (yes or no); 4) early-life postnatal exposures measured at 9 months: breastfeeding (never, $\leqslant 1$ week, 1-6 weeks, 6 weeks-6 months, $>6$ months), parental smoking in the same room (yes or no), damp/ condensation in the home (yes or no), exposure to "pollution, grime or other environmental problems" fairly or very commonly in the residential area (yes or no, based on maternal report), furry pets in household (yes or no) and number of siblings in household and childcare use (informal (e.g. family members) and formal (e.g. childminder, crèche or nursery)) to capture potential exposure to infections.

\section{Analysis strategy}

First, we described the prevalence of wheezing phenotypes, overall and according to maternal education (the primary exposure of interest). Next, all other risk factors were stratified by maternal education. Relative risk ratios (RRRs) and 95\% confidence intervals for early and persistent wheeze were estimated in multinomial regression models, according to maternal education, unadjusted and then sequentially adjusting for the other risk factors. In the sequential models we calculate adjusted RRRs for wheezing patterns on the basis of maternal education (with children of mothers with degree-level qualifications as the reference group), mutually adjusting for the other early-life risk factors. Taking a life-course approach [20], these risk factors were entered into the model in the chronological order in which they were experienced: first we adjusted for demographic factors, then maternal characteristics fixed before birth, then exposures during pregnancy and perinatal exposures and finally postnatal exposures, to show the impact on the association between (SECs) and asthma symptoms. Any observed change in RRRs in a final complete case sample was taken to indicate potential mediation (fig. 1) [21]. We visualised the change in RRR comparing mothers with no qualifications to those with the highest education (the SEC gap [22]). Analyses were conducted using Stata/SE (version 13; Stata Corp., College Station, TX, USA) with svy commands to account for the sampling design and attrition using survey weights. We estimated all model parameters using the maximum likelihood. We undertook a range of sensitivity analyses, repeating the analysis with alternative measures of SECs (parental occupational social class), including children with more severe wheezing and repeating the analysis stratified on the basis of eczema status at 9 months.

\section{Results}

Of the 11538 MCS children who were present at all four data sweeps, 11418 (99\%) had full data on wheezing. 11141 children had data on both wheezing and maternal education (96\%), and 10251 (89\%) had full data on all exposures of interest. Adjusted for the sampling design and attrition, 66\% (7540) never had wheezing, $17.1 \%(\mathrm{n}=1948)$ were classified as having early remitting wheeze, 12\% (1393) had persistent/relapsing wheeze, and 4.9\% (537) had transient/intermittent wheezing. Table 1 shows the

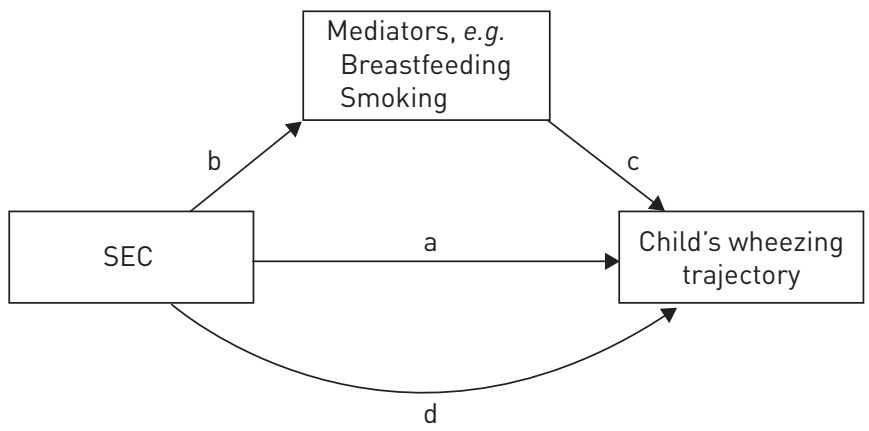

FIGURE 1 The four stages of the analysis to test mediators of the association between socioeconomic circumstances (SECs) (parental education) and wheezing. a) Estimate unadjusted risk ratio by SECs, also adjusted for demographic factors, e.g. sex and ethnicity; b) explore association between SECs and hypothetical mediators; cl explore association between hypothetical mediators and wheezing; d) enter hypothetical mediators into model; mediation taken to be a reduction in relative risk ratio. 
TABLE 1 Characteristics of the total study population, by maternal qualifications

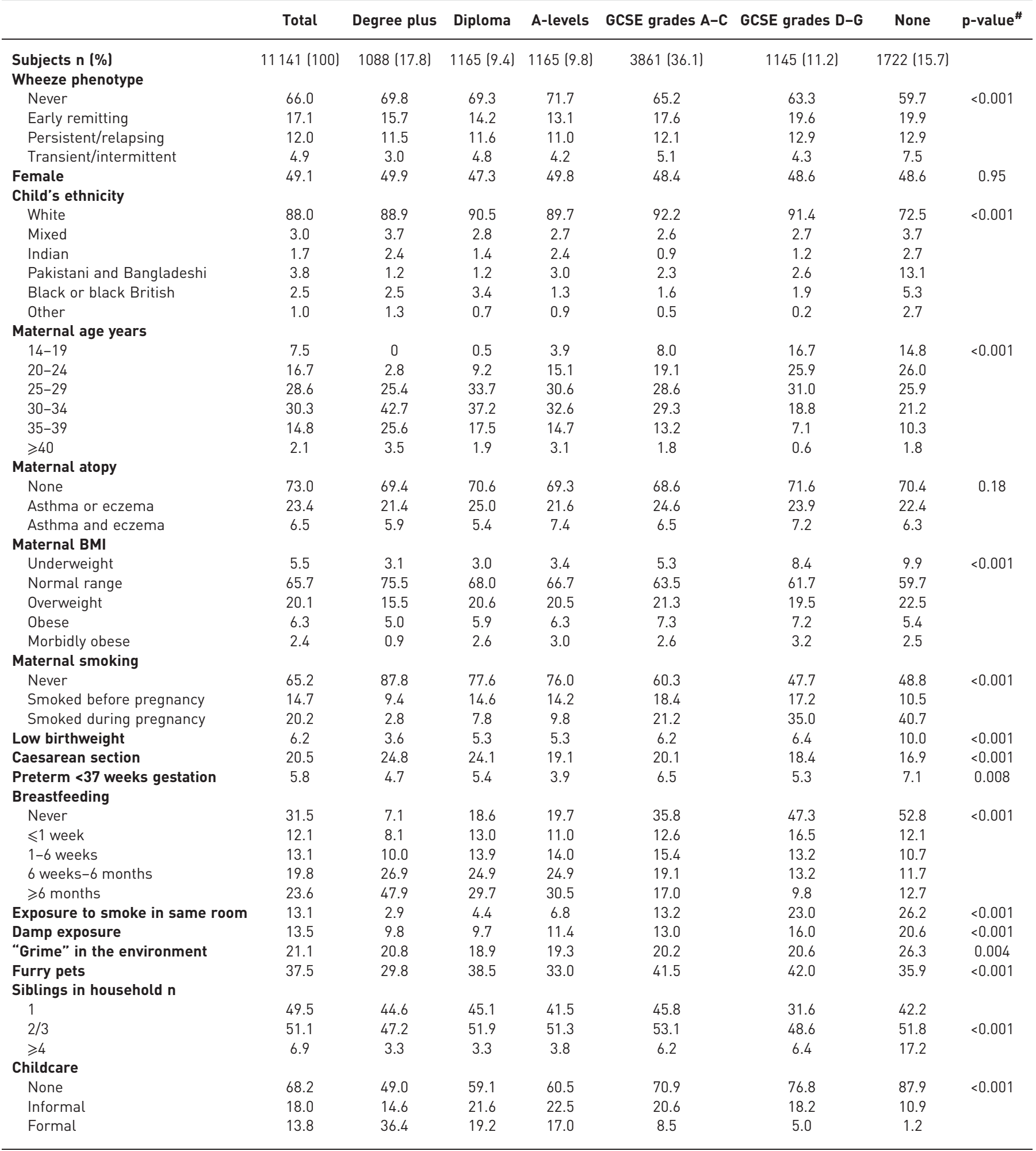

Data are presented as \%, unless otherwise stated. All percentages are adjusted for sampling design and attrition. Item missingness: ethnicity 23, maternal age 7, maternal atopy 17, maternal body mass index (BMI) 657, maternal smoking 36, preterm birth 155, Caesarean section 9, environmental tobacco smoke exposure 1, damp exposure 16, grime exposure 103, furry pet exposure 1, childcare type 42. GCSE: General Certificate of Secondary Education. " : Chi-squared. 
characteristics of the population stratified by maternal qualifications. The prevalence of wheezing is lower in children whose parents have higher educational qualifications. Educational differences were present in all other covariates of interest, except for sex and maternal atopy.

\section{Association of covariates with wheezing phenotypes}

In univariate multinomial regression, with never wheezing as the reference group, lower maternal qualifications, male sex, maternal overweight/obesity, maternal atopy, prematurity, maternal smoking, never having breastfed and postnatal smoke exposure were associated with an increased RRR for both early and persistent wheezing (table 2).

Maternal atopy was associated with persistent/relapsing wheezing and early remitting wheezing, although the RRR was greater for the former. High and low maternal age at childbirth were associated with early remitting wheezing, but there was no relationship with persistent/relapsing wheeze. Smoking before pregnancy but then giving up during pregnancy conveyed an increased risk of both early and persistent/ relapsing wheezing, but the RRR was larger for smoking during pregnancy. Furthermore, there was evidence of a dose-response relationship with the number of cigarettes smoked per day during pregnancy. Caesarean section was associated with a greater risk of persistent/relapsing wheeze, but not early wheezing. Longer duration of breastfeeding was associated with a reduction in the risk of both types of wheezing. High and low maternal age at the birth of the cohort child, environmental tobacco smoke exposure, damp and "grime" exposure at 9 months were associated with an increased risk of early remitting wheeze, but not persistent/relapsing wheeze.

\section{Association between maternal education and wheezing, adjusted for other early-life factors}

Figure 2 explores the extent to which the elevated RRR of wheeze in children of mothers with no educational qualifications (compared to those with a degree) is attenuated after adjustment for other covariates, and shows the RRRs for early wheeze and persistent/relapsing wheeze after adjustment for covariates, added sequentially (the online supplementary material includes data tables showing all the model coefficients, across the entire socioeconomic gradient). The increased risk of both wheezing types in children whose mothers had no qualifications remains after adjusting for sex and ethnicity. Adjusting for maternal age led to a partial reduction in the RRR for early wheeze (from 1.53 (95\% CI 1.26-1.86) to 1.34 (95\% CI 1.10-1.62)), but not for persistent/relapsing wheeze, and subsequent adjustment for maternal atopy and BMI did not change the RRR. Smoking during pregnancy reduced the RRR substantially for both wheezing types (1.12 (95\% CI 0.91-1.37) for early wheeze and 1.13 (95\% CI $0.83-1.52$ ) for persistent/ relapsing wheeze). Further adjustment for perinatal factors had no additional effect on the point estimates, whereas further adjusting for breastfeeding reduced both effect estimates to $\sim 1$. In a post hoc analysis fitting a model adjusting only for smoking during pregnancy and breastfeeding completely removed the educational inequalities for both phenotypes. By contrast, adjusting only for postnatal environmental influences (e.g. environmental smoke exposure, damp, etc.) did not reduce the RRR for early wheeze, and led to a small reduction in the RRR for persistent/relapsing wheeze (online supplementary table S3).

\section{Adjusted association between risk factors and wheezing phenotypes}

Figure 3 shows the covariate estimates for the fully adjusted model (online supplementary material). Female sex was significantly protective for both wheezing phenotypes in all of the models, but more so for persistent/ relapsing wheeze compared to early wheeze. Both low and high maternal age were associated with increased risk of early wheeze in the fully adjusted model, but low maternal age was protective for persistent/relapsing wheeze in the fully adjusted model. The effect of maternal atopy was greater in the persistent/relapsing wheezing group, in contrast to the early wheezing phenotype, and there was a trend towards increased risk of both wheezing phenotypes with higher maternal BMI. Preterm delivery was the only perinatal factor that remained significant in the fully adjusted model, increasing the risk of both wheezing phenotypes. Exposure to two or three siblings (but not four or more siblings) and childcare increased the risk of early remitting wheezing, whereas furry pet exposure decreased the risk of persistent/relapsing asthma.

\section{Sensitivity analyses}

The conclusions of the study were similar when we used parental occupational social class; when we used more severe wheezing as the outcome; and in children without eczema at 9 months. In children with eczema there was no clear association between maternal education and wheezing phenotypes, but the sample was small with wide confidence intervals (online supplementary material).

\section{Discussion}

Using a nationally representative sample of UK children we show that early transient and persistent/ relapsing patterns of wheezing are common and socially patterned. Male sex, maternal age at birth of child, 
TABLE 2 Prevalence of wheeze patterns by covariates and univariate relative risk ratios (RRRs) for wheeze patterns for each covariate

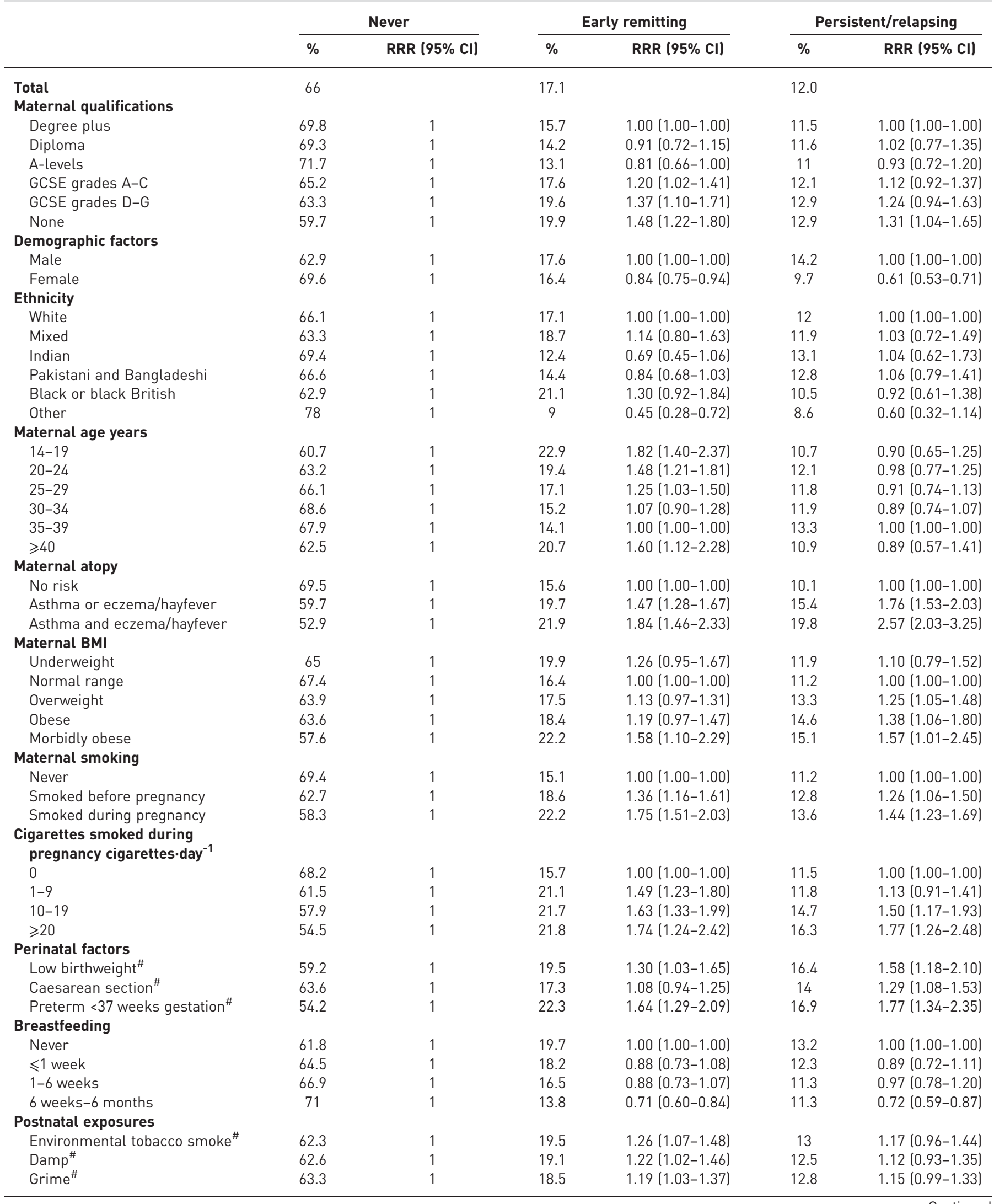


TABLE 2 Continued

\begin{tabular}{|c|c|c|c|c|c|c|}
\hline & \multicolumn{2}{|c|}{ Never } & \multicolumn{2}{|c|}{ Early remitting } & \multicolumn{2}{|c|}{ Persistent/relapsing } \\
\hline Pets ${ }^{\#}$ & 66.3 & 1 & 16.9 & $0.99(0.89-1.11)$ & 11.3 & $0.90(0.79-1.04)$ \\
\hline 1 & 66.5 & 1 & 16.4 & $1.00(1.00-1.00)$ & 11.9 & $1.00(1.00-1.00)$ \\
\hline $2 / 3$ & 65.8 & 1 & 17.9 & $1.10(0.98-1.24)$ & 11.8 & $1.00(0.89-1.12)$ \\
\hline$\geqslant 4$ & 66.5 & 1 & 13.9 & $0.85(0.66-1.09)$ & 14.4 & $1.21(0.93-1.56)$ \\
\hline Informal & 66.3 & 1 & 17.7 & $1.06(0.91-1.24)$ & 4.4 & $0.94(0.79-1.12)$ \\
\hline Formal & 67.6 & 1 & 18.3 & $1.08(0.93-1.24)$ & 3.5 & $0.84(0.68-1.04)$ \\
\hline
\end{tabular}

Data are presented as \% or RRR $(95 \% \mathrm{CI}) . \mathrm{n}=11141$. GCSE: General Certificate of Secondary Education; BMI: body mass index. ${ }^{\#}$ : reference group for binary variables not shown; reference not low birthweight/Caesarean section/preterm birth.

BMI, atopy, smoking during pregnancy, preterm birth, breastfeeding, exposure to other children and furry pets were independently associated with wheezing, but the strength and pattern of association varied between wheezing types. Children of mothers with no educational qualifications were $\sim 40 \%$ more likely to have transient wheezing in the early years of life, and $\sim 30 \%$ more likely to have wheezing that persists to the age of 7 years. Despite the social patterning of many of the risk factors for asthma symptoms, the social differences in wheezing were largely attenuated by smoking during pregnancy, which significantly increases the risk of wheezing in a dose-dependent manner; and breastfeeding, which is protective in a dose-dependent manner. Therefore, our data suggest that policies to reduce the social inequalities in these factors are likely to reduce inequalities in wheezing and asthma.

\section{Comparison with other findings}

The dose-response relationships with smoking during pregnancy and breastfeeding, and the increased risk associated with preterm birth were the same for both wheezing types, and corroborate recent large meta-analyses of epidemiological studies [23-26]. A systematic review of observational studies suggests a protective effect of breastfeeding on wheezing, but this has not been shown in intervention trials [24, 27].

Other risk factors identified in this study varied for early and persistent/relapsing wheezing patterns. As in the Tucson Children's Respiratory Study early transient wheezing was associated exposure to other siblings and childcare, which increase the risk of early respiratory infection [28, 29]. Furthermore, persistent wheeze was more strongly associated with male sex and maternal atopy [14]. There is considerable debate around the effect of pet exposure on wheezing and asthma risk. A recent pooled analysis of cohort studies suggested no overall effect [30], but in the MCS a significant protective effect emerges in the adjusted model for persistent/relapsing wheezing. Previous studies have demonstrated contrasting associations between wheezing and maternal age at delivery [31]. Most have shown an inverse association of asthma symptoms with maternal age [31,32], but some studies have demonstrated the opposite [33]. Furthermore, U-shaped associations between maternal age and atopy risk have been described [31]. These differences may be partially explained by the complex pattern of association revealed in our study. Younger maternal age was protective for persistent/relapsing wheezing, but possibly associated with increased risk of early wheezing after adjusting for a large range of confounding factors, including perinatal factors and SECs. It remains unclear whether these associations are driven by the biological ageing of the mother, by residual sibling effects or by other confounding factors.

Our study is one of the largest to explore inequalities in longitudinal wheezing patterns in children, and the first to demonstrate that adjusting for maternal age, maternal smoking and breastfeeding attenuates all the socioeconomic difference in the prevalence of common wheezing patterns. Most previous studies have shown that wheezing is more common in more disadvantaged children [5], but only a few have explored potentially mediating pathways. Violato et al. [17] examined income inequalities in respiratory health in the UK MCS up to the age of 5 years, and concluded that household income has an effect on wheezing after controlling for potential mechanisms that mediate the income-child health association. A recent study used a data-driven latent-class analysis approach to explore risk factors for atopy trajectories (wheeze and other atopic symptoms) in the MCS [34]. The authors identified household damp and breastfeeding initiation as potentially modifiable risk factors, but did not explicitly assess how social inequalities in wheezing are mediated. Two Dutch studies examined risk factors for asthma symptoms at different ages throughout 


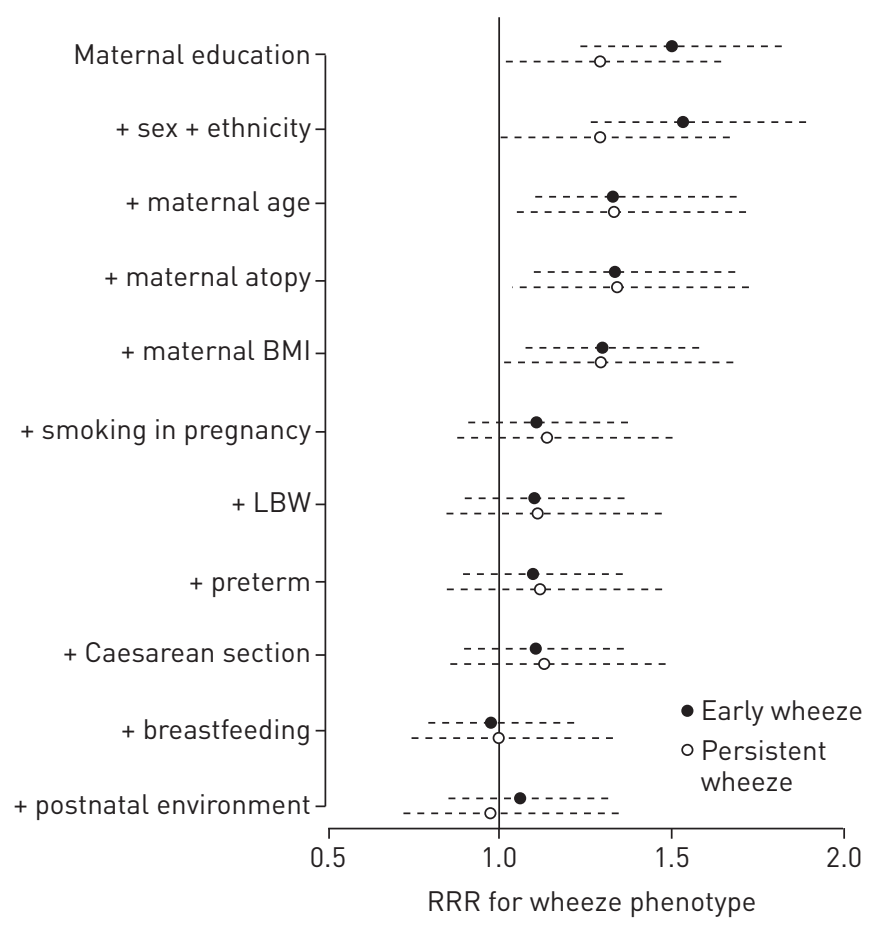

FIGURE 2 Relative risk ratios (RRRs) for wheezing phenotypes comparing the lowest maternal education group to the highest in sequentially adjusted models. The final adjusted model includes maternal education + sex + ethnicity + maternal age at birth of child + maternal atopy + smoking in pregnancy + low birthweight $($ LBW $)+$ preterm birth + Caesarean section + breastfeeding + postnatal environment (environmental tobacco smoke, "grime", damp, pets, siblings and childcare). BMI: body mass index.

childhood in cross-sectional analyses of cohort data, but since neither of these studies examined longitudinal patterns of wheeze it is not possible to compare findings directly, although there were some similarities in findings, with breastfeeding and smoking explaining some of the inequality in wheezing $[18,21]$.

\section{Strengths and limitations}

This study used secondary data from a large and contemporary UK cohort and the results are likely to be generalisable to other high-income countries. A wide range of information is collected in the MCS, which allowed us to explore demographic, environmental and perinatal risk factors for wheezing, including different measures of SECs. A limitation of the study is that wheezing status was based upon maternal report, rather than a clinical diagnosis of asthma. However, we were able to make use of the validated asthma questionnaire from the ISAAC questionnaires [11], used in the MCS. There is much debate in the paediatric literature about how best to classify childhood wheezing [35]. We intended to focus on the most common phenotypes at a population level, and those of greatest public health significance. To do this we followed the approaches used in other studies to distinguish empirically early remitting and persistent/ relapsing wheezing pattern $[13,14]$. We note that other studies have used data-driven latent-class approaches to classify wheezing patterns $[29,36]$. It is reassuring that the risk factors for wheezing patterns identified corroborate previous findings, and that similar conclusions were reached in our study when the analysis was restricted to patients with more severe asthma symptoms (more than four episodes per year).

Attrition is a ubiquitous problem in cohort studies, and is a potential source of bias. $62 \%$ of the children who were originally recruited at 9 months were included in this analysis. However, one advantage of the MCS is the use of sampling and response weights, which account to some extent for the attrition in the dataset. Of the children present at all sweeps, $11 \%$ had some missing data on one or more of the covariates in the analysis. A complete-case analysis was used, whereby these individuals were excluded from the analysis. This approach can be inefficient, which is a problem with smaller datasets, and may also introduce bias, when the individuals who are excluded are not a random sample from the target population. However, in this analysis the sample was large, and the internal associations, which were the targets of inference within the sample population, are likely to be valid, but we speculate that they may underestimate the effect sizes in the full UK population. Despite the large number of variables collected in the MCS, not all of the exposures pertinent to the study of asthma are collected. For example, air pollution is important in the aetiology of asthma, but no specific data are collected on this in MCS. 

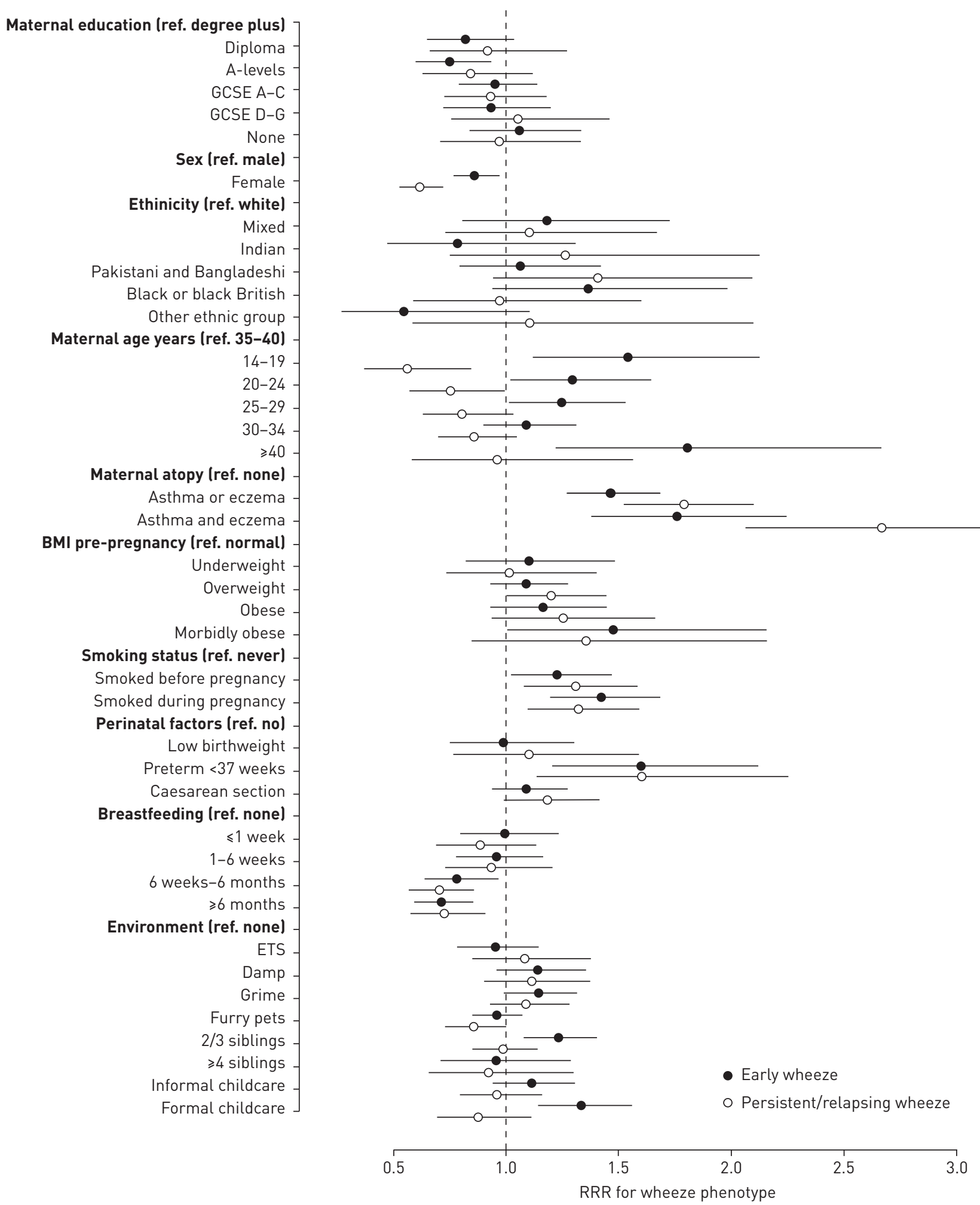

FIGURE 3 Fully adjusted associations between covariates and wheezing phenotypes. GCSE: General Certificate of Secondary Education; BMI: body mass index; ETS: environmental tobacco smoke; RRR: relative risk ratio.

Policy and practice implications

The UK has the highest prevalence of asthma symptoms internationally [37], and this study confirms important differences in the prevalence of wheezing symptoms by maternal educational level in the UK, and provides data on the most appropriate targets for public health intervention. We encourage future studies using alternative 
methods for causal inference in longitudinal data [38] to further unravel the causal pathways involved in the development of inequalities in wheezing outcomes. Parental atopy and maternal age are important risk factors for wheezing, but are less amenable to public health intervention. Stopping smoking during pregnancy and promoting breastfeeding have long been recognised as important targets for child health promotion, but it is clear that much more needs to be done to reduce the large social differentials in these key mediating factors. Table 1 shows that $40 \%$ of women with no qualifications in the UK smoke during pregnancy, compared to $2.8 \%$ of women with degrees. The figures are similar for breastfeeding. Early identification of pregnant women who smoke, and appropriate counselling and referral to smoking cessation services need to be intensified for women living in more disadvantaged conditions, along with breastfeeding support programmes. The long-term solution to health inequalities in asthma is likely to be one that takes broader action to address the social determinants of health and our analysis provides further evidence that the antenatal period and early years represent important periods for targeting interventions to reduce inequalities [39].

\section{References}

1 Carson C, Sacker A, Kelly Y, et al. Asthma in children born after infertility treatment: findings from the UK Millennium Cohort Study. Hum Reprod 2013; 28: 471-479.

2 Patel SP, Järvelin MR, Little MP. Systematic review of worldwide variations of the prevalence of wheezing symptoms in children. Environ Health 2008; 7: 57.

3 Sennhauser FH, Braun-Fahrländer C, Wildhaber JH. The burden of asthma in children: a European perspective. Paediatr Respir Rev 2005; 6: 2-7.

4 Asthma UK. Asthma Facts and Statistics. www.asthma.org.uk/asthma-facts-and-statistics Date last accessed: April 21, 2013.

5 Uphoff E, Cabieses B, Pinart M, et al. A systematic review of socioeconomic position in relation to asthma and allergic diseases. Eur Respir J 2015; 46: 364-374.

6 Lodge CJ, Lowe AJ, Allen KJ, et al. Childhood wheeze phenotypes show less than expected growth in FEV 1 across adolescence. Am J Respir Crit Care Med 2014; 189: 1351-1358.

7 Lowe LA, Simpson A, Woodcock A, et al. Wheeze phenotypes and lung function in preschool children. Am J Respir Crit Care Med 2005; 171: 231-237.

8 Subbarao P, Mandhane PJ, Sears MR. Asthma: epidemiology, etiology and risk factors. CMAJ 2009; 181: E181-E190.

9 Illi S, von Mutius E, Lau S, et al. Perennial allergen sensitisation early in life and chronic asthma in children: a birth cohort study. Lancet 2006; 368: 763-770.

10 Connelly R, Platt L. Cohort profile: UK Millennium Cohort Study (MCS). Int J Epidemiol 2014; 43: 1719-1725.

11 Asher I. ISAAC International Study of Asthma and Allergies in Childhood. Pediatr Pulmonol 2007; 42: 100.

12 Martinez FD, Wright AL, Taussig LM, et al. Asthma and wheezing in the first six years of life. The Group Health Medical Associates. N Engl J Med 1995; 332: 133-138.

13 Stein RT, Holberg CJ, Morgan WJ, et al. Peak flow variability, methacholine responsiveness and atopy as markers for detecting different wheezing phenotypes in childhood. Thorax 1997; 52: 946-952.

14 Stein RT, Martinez FD. Asthma phenotypes in childhood: lessons from an epidemiological approach. Paediatr Respir Rev 2004; 5: 155-161.

15 Henderson J, Granell R, Heron J, et al. Associations of wheezing phenotypes in the first 6 years of life with atopy, lung function and airway responsiveness in mid-childhood. Thorax 2008; 63: 974-980.

16 Savenije OE, Granell R, Caudri D, et al. Comparison of childhood wheezing phenotypes in 2 birth cohorts: ALSPAC and PIAMA. J Allergy Clin Immunol 2011; 127: 1505-1512.

17 Violato M, Petrou S, Gray R. The relationship between household income and childhood respiratory health in the United Kingdom. Soc Sci Med 2009; 69: 955-963.

18 Ruijsbroek A, Wijga AH, Kerkhof M, et al. The development of socio-economic health differences in childhood: results of the Dutch longitudinal PIAMA birth cohort. BMC Public Health 2011; 11: 225.

19 Hafkamp-de Groen E, Sonnenschein-van der Voort AM, Mackenbach JP, et al. Socioeconomic and sociodemographic factors associated with asthma related outcomes in early childhood: the Generation R Study. PLoS One 2013; 8: e78266.

20 Carson C, Kelly Y, Kurinczuk JJ, et al. Effect of pregnancy planning and fertility treatment on cognitive outcomes in children at ages 3 and 5: longitudinal cohort study. BMJ 2011; 343: d4473.

21 Hafkamp-de Groen E, van Rossem L, de Jongste JC, et al. The role of prenatal, perinatal and postnatal factors in the explanation of socioeconomic inequalities in preschool asthma symptoms: the Generation R Study. J Epidemiol Community Health 2012; 66: 1017-1024.

22 Graham H. Tackling inequalities in health in England: remedying health disadvantages, narrowing health gaps or reducing health gradients? J Soc Pol 2004; 33: 115-131.

23 Neuman A, Hohmann C, Orsini N, et al. Maternal smoking in pregnancy and asthma in preschool children: a pooled analysis of eight birth cohorts. Am J Respir Crit Care Med 2012; 186: 1037-1043.

24 Dogaru CM, Nyffenegger D, Pescatore AM, et al. Breastfeeding and childhood asthma: systematic review and meta-analysis. Am J Epidemiol 2014; 179: 1153-1167.

25 Sonnenschein-van der Voort AM, Arends LR, de Jongste JC, et al. Preterm birth, infant weight gain, and childhood asthma risk: a meta-analysis of 147,000 European children. J Allergy Clin Immunol 2014; 133: 1317-1329.

26 Been JV, Lugtenberg MJ, Smets E, et al. Preterm birth and childhood wheezing disorders: a systematic review and meta-analysis. PLoS Med 2014; 11: e1001596.

27 Kramer MS, Kakuma R. Optimal duration of exclusive breastfeeding. Cochrane Database Syst Rev 2012; 8: CD003517.

28 Ball TM, Castro-Rodriguez JA, Griffith KA, et al. Siblings, day-care attendance, and the risk of asthma and wheezing during childhood. N Engl J Med 2000; 343: 538-543. 
Granell R, Sterne JA, Henderson J. Associations of different phenotypes of wheezing illness in early childhood with environmental variables implicated in the aetiology of asthma. PLoS One 2012; 7: e48359.

30 Lødrup Carlsen KC, Roll S, Carlsen KH, et al. Does pet ownership in infancy lead to asthma or allergy at school age? Pooled analysis of individual participant data from 11 European birth cohorts. PLoS One 2012; 7: e43214.

31 Laerum BN, Svanes C, Wentzel-Larsen T, et al. Young maternal age at delivery is associated with asthma in adult offspring. Respir Med 2007; 101: 1431-1438.

32 Australian Centre for Asthma Monitoring. Asthma in Australian Children: Findings from Growing Up in Australia, the Longitudinal Study of Australian Children. www.aihw.gov.au/publication-detail/?id=6442468289 Date last accessed: September 4, 2014. Date last updated: October 14, 2009.

33 Dik N, Tate RB, Manfreda J, et al. Risk of physician-diagnosed asthma in the first 6 years of life. Chest 2004; 126: 1147-1153.

34 Panico L, Stuart B, Bartley M, et al. Asthma trajectories in early childhood: identifying modifiable factors. PLoS One 2014; 9: e111922.

35 Spycher BD, Silverman M, Kuehni CE. Phenotypes of childhood asthma: are they real? Clin Exp Allergy 2010; 40: 1130-1141.

36 Savenije OE, Kerkhof M, Reijmerink NE, et al. Interleukin-1 receptor-like 1 polymorphisms are associated with serum IL1RL1-a, eosinophils, and asthma in childhood. J Allergy Clin Immunol 2011; 127: 750-756.

37 Worldwide variation in prevalence of symptoms of asthma, allergic rhinoconjunctivitis, and atopic eczema: ISAAC. The International Study of Asthma and Allergies in Childhood (ISAAC) Steering Committee. Lancet 1998; 351: 1225-1232.

38 Richiardi L, Bellocco R, Zugna D. Mediation analysis in epidemiology: methods, interpretation and bias. Int J Epidemiol 2013; 42: 1511-1519.

39 CSDH. Closing the gap in a generation: health equity through action on the social determinants of health. Final Report of the Commission on Social Determinants of Health. http://whqlibdoc.who.int/publications/2008/ 9789241563703_eng.pdf Date last accessed: August 29, 2008. Date last updated: 2008. 